\title{
Achromatic Fresnel Lens with Improved Efficiency for PV Systems
}

\author{
Mario González Montes, ${ }^{1}$ Juan Carlos Martínez-Antón, ${ }^{1}$ Daniel Vázquez Moliní, ${ }^{1}$ \\ Antonio Álvarez Fernandez-Balbuena, ${ }^{1}$ and E. Bernabeu ${ }^{2}$ \\ ${ }^{1}$ Applied Optics Complutense Group, Universidad Complutense de Madrid, Arcos del Jalón 118, 28037 Madrid, Spain
${ }^{2}$ Applied Optics Complutense Group, Universidad Complutense de Madrid, Facultad de Ciencias Físicas, Ciudad Universitaria,
Plaza Ciencias, s/n, 28040 Madrid, Spain
}

Correspondence should be addressed to Mario González Montes; mariog@fis.ucm.es

Received 18 September 2013; Accepted 29 January 2014; Published 12 March 2014

Academic Editor: Xie Quan

Copyright (c) 2014 Mario González Montes et al. This is an open access article distributed under the Creative Commons Attribution License, which permits unrestricted use, distribution, and reproduction in any medium, provided the original work is properly cited.

\begin{abstract}
This work is aimed to design and evaluate different achromatic Fresnel lens solutions capable of operating as concentrators aimed at photovoltaic cells systems. Throughout this study, the theoretical parametric design of the achromatic lens will be shown together with a series of simulations to verify the performance of each lens topology. The results will be compared with a standard Fresnel lens to ascertain the validity and effectiveness of the obtained design. Finally, a novel kind of hybrid lens is proposed, which combines the advantages of each type of lens (standard and Fresnel) according to the optimal operating region of each design. Efficiency and concentration ratios of each particular lens are shown, regarding lens dimension, light's incidence angle, or wavelength. Through this innovative achromatic design concentration ratios above 1000 suns, which hardly reach standard Fresnel lenses. Furthermore chromatic dispersion is minimized and the efficiency rate is over $85 \%$ of efficiency for a wide spectral range (from $350 \mathrm{~nm}$ to $1100 \mathrm{~nm})$.
\end{abstract}

\section{Introduction}

An achromatic lens is designed to reduce the chromatic aberrations inherent in standard lenses. Achromatic lenses are designed for a range of wavelengths to focus a parallel light beam on a converging point located at the focal distance with the minimum chromatic aberrations. The most common type is the achromatic doublet based on two individual lenses, one crown glass lens element and a flint glass lens element, joined together, usually one positive and the other negative, so the aberrations and dispersion effects of one lens are compensated by those of the other [1]

According to Lorenzo [2] to design a low or moderate concentration system the chromatic aberrations are not very important but when designing high concentration $\mathrm{PV}$ systems the effect of chromatic aberration will be very important.

Achromatic system designs are known since XVIII century when Dollond made a first system with glass and water lenses. The design of a double prism with two different materials with achromatic properties is also much known $[3,4]$. Achromatic lenses are usually found as holographic or phase design technologies to reduce the effects of chromatic aberrations on final quality $[5,6]$.

This effect appears not only in classical but also in nonimaging Fresnel lenses. Chromatic aberrations in Fresnel lenses working as photovoltaic (PV) primary optics concentrators are an important limit in order to obtain high concentration ratios [2], leading to new optical solutions for the primary concentration system as aplanatic lens [7], low dispersion glasses, and achromatic doublets [8]. The maximum theoretical concentration factor for standard Fresnel lens is typically limited about 1000x at normal incidence while refractive doublets present a smaller aberration increasing the concentration factor up to 2000x. Recently a 2000x factor has been experimentally measured for a flat achromatic Fresnel doublet [8].

This study aims to develop an achromatic lens design that combines the advantages of Fresnel lenses (high efficiency, 
reduced thickness, and lightweight construction) along with the stability and lack of spectrum aberrations of achromatic lenses. The achromatic doublet design will be carried out by means of a parametric analysis to reject the less efficient configurations, thus leading to a set of optimal solutions that will be compared in terms of efficiency, stability flux distribution, uniformity, and so forth.

There will be provided innovative hybrid designs that exploit optimal operating areas of each lens topology and a thorough analysis will be performed taking into account the performance of each design regarding the incidence angle of the solar light and its spectrum.

\section{System's Description}

Throughout this work a set of comparisons will be carried out among standard Fresnel lens, considering the flat facets Fresnel lens, in opposition to a number of own design achromatic doublets which are formed by following materials: polymethylmethacrylate (PMMA), which is one of most employed plastics in solar concentration applications due to its high transmittance and low dispersion curve, and polycarbonate (PC), which has similar spectral transmission and high impact resistance [9]. Therefore conventional achromatic doublets composed by these same materials are quite common [10], due to their low cost and suitable refractive behavior (high refractive index difference seen in Figure 1). PMMA lenses, at the ease and economic feasibility of fabrication, have low mechanical rigidity and stability of optical characteristics at extended operation in the conditions of the effect of the environment factors.

Fresnel Lenses are formed by generating grooves onto the surface of a plastic plate and appropriately tiling the optical surface within each groove to form a narrow annular pattern. Fresnel lenses are manufactured by means of direct copying the Fresnel profile from a negative mold on glass during ultraviolet polymerization or photolithography techniques using a large mask that matches the designed pattern.

The design of Fresnel lenses is subject to certain limitations due to the manufacturing molding process which implies tooth peaks deviations of about $1-2 \mu \mathrm{m}$ [11] and inaccuracies rounded teeth [12]. Recently these processes have been greatly improved due to improvements in manufacturing processes such as cooling temperature control techniques as air flow cooling [13]. Nowadays commercial Fresnel lenses manufactured for solar concentration purpose reach a maximum dimension of about $150 \times 100 \mathrm{~cm}$ and assure an average efficiency of $82 \%$ and concentration ratios close to $800 x$ [11].

Most of Fresnel lenses are manufactured with glass; one of the main disadvantages of glass as a Fresnel manufacturing material is the limitation to obtain an operating profile, for mass production (e.g., injection molding), of high power efficiency. This difficulty is caused by its inflexibility to reduce the profile pitch, which can be of about $0.3 \mathrm{~mm}$ in modern polymer Fresnel lens.

In order to characterize the materials employed in the achromatic doublet, the corresponding behavior of the refractive index versus wavelength of incident light is shown

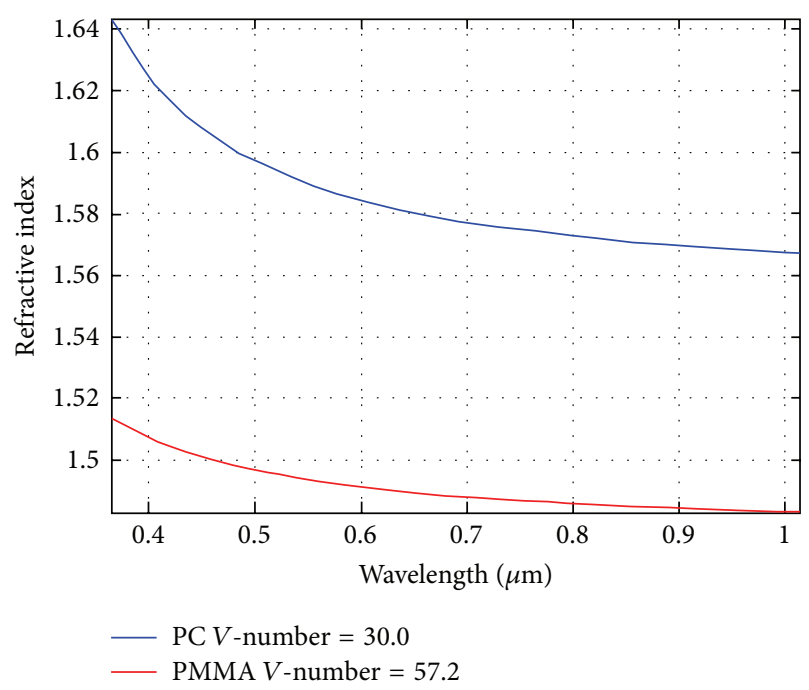

FIgUre 1: Refractive index of PC on PMMA.

in Figure 1. The Abbe number $(V)$ is also calculated and gives us an amount of the level of dispersion of these materials; the higher the value of this parameter, the better the quality of the lens, but a commitment to the manufacturing cost of the doublet is also required.

Abbe numbers are used to calculate the necessary focal lengths of achromatic doublet lenses to minimize chromatic aberration but Abbe numbers do not give real information about the quality of the achromatization. In order to develop this study, as a first step, we have selected a configuration for the PV system which can be found in the PV (photovoltaic) market, with a focal distance of $155 \mathrm{~mm}$ and a numerical aperture NA $=0.38$; therefore its radius is $120 \mathrm{~mm}$. At the focal point the secondary optics of BK7 glass are located with prismatic geometry as we show in Figure 2 . The first surface of the prismatic secondary optics is a square of $11 \times 11 \mathrm{~mm}$, the exit surface is a square of $5.5 \times 5.5 \mathrm{~mm}$, and its height is $38 \mathrm{~mm}$ shown in Figure 2.

The shape of the secondary reflector should be directly related to the primary concentrator (the achromatic lens) because it works on the previously deflected rays. A wide range of different designs of these elements can be found; however the most popular ones can be classified in the following groups: domed shapes, CPCs, and truncated pyramids or cones [14].

The achromatic doublet lens is composed of PC in its upper surface and it is completed with PMMA in its lower surface as we show in Figure 3. Based on the standard achromatic doublet widely discussed in the literature [15], a Fresnel format doublet, composed of pair different refractive index plastic materials, will be designed. In order to avoid losses due to accumulation of waste and dirt the upper surface of the lens must be plane. Therefore the achromatic doublet design must be composed of two phases: the first one consists of a double saw PMMA profile and the second phase is made of a PC layer as described in Figure 3.

Along this section, following the Fermat's principle (Snell equations), an innovative Fresnel achromatic doublet is 


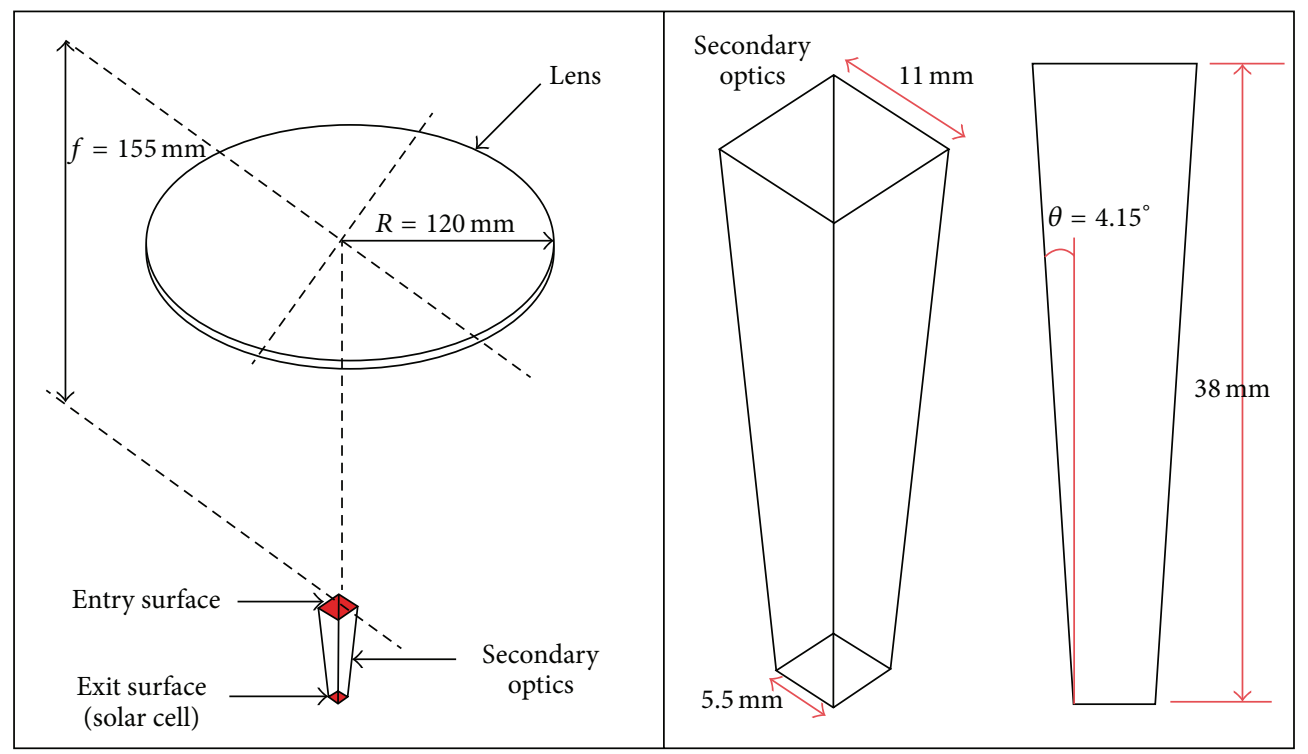

FIGURE 2: Optics sketch of the studied system.

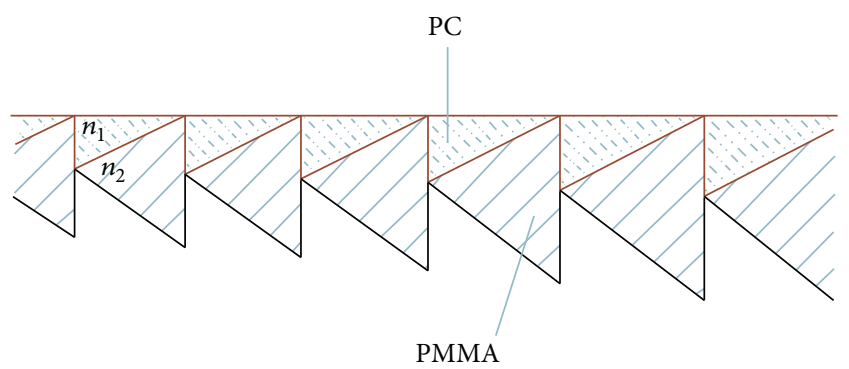

FIGURE 3: Section of the achromatic Fresnel lens.

proposed (hereafter mentioned as achromatic lens) which forms the core of the following development and simulations in the present paper. Next the mathematical development that leads to the achromatic lens design will be shown (Figure 4).

Consider the Snell equations:

$$
\begin{gathered}
n_{1} \sin \left(\varepsilon_{1}\right)=n_{2} \sin \left(\varepsilon_{2}\right), \\
n_{2} \sin \left(\varepsilon_{3}\right)=\sin \left(\varepsilon_{4}\right) .
\end{gathered}
$$

Also consider the incidence and transmission angles:

$$
\varepsilon_{1}=\alpha_{1} .
$$

Restating Snell equations,

$$
\varepsilon_{2}=\sin ^{-1}\left(\frac{n_{1}}{n_{2}} \sin \left(\varepsilon_{1}\right)\right) .
$$

By the properties of any triangle,

$$
\varepsilon_{2}+\varepsilon_{3}+\left(180-\alpha_{2}\right)=180 \Longrightarrow \varepsilon_{3}=\alpha_{2}-\varepsilon_{2} .
$$

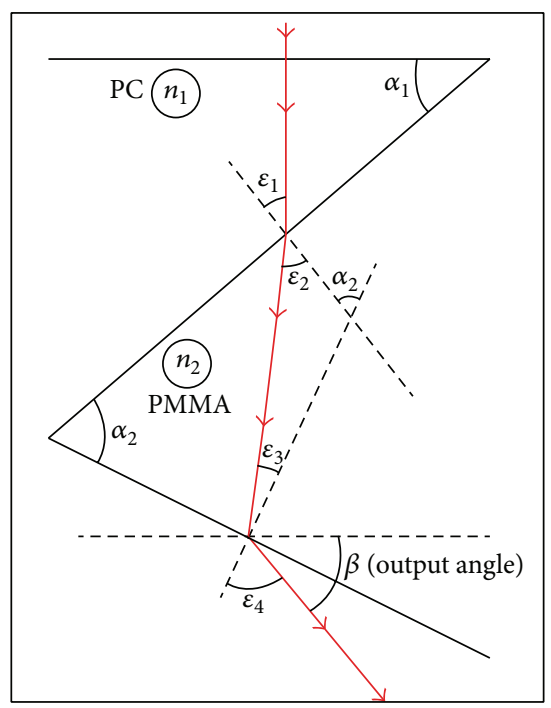

Figure 4: Ray refraction across achromatic lens.

Substituting (3) and (4) in the restated $\varepsilon_{4}$ Snell equation,

$$
\begin{aligned}
\varepsilon_{4} & =\sin ^{-1}\left(n_{2} \sin \left(\varepsilon_{3}\right)\right)=\sin ^{-1}\left(n_{2} \sin \left(\alpha_{2}-\varepsilon_{2}\right)\right) \\
& =\sin ^{-1}\left(n_{2} \sin \left(\alpha_{2}-\sin ^{-1}\left(\frac{n_{1}}{n_{2}} \sin \left(\varepsilon_{1}\right)\right)\right)\right) .
\end{aligned}
$$

Output Angle. Finally the output angle of the light beam passing through the achromatic lens can be expressed as a function depending only on the prisms (PC and PMMA) angles $\left[\alpha_{1}, \alpha_{2}\right]$ and their respective refractive index $\left[n_{1}, n_{2}\right]$ 
by simply replacing the output angle's components by (2), (4), and (5):

$$
\begin{aligned}
\beta= & 90^{\circ}+\left(\varepsilon_{2}-\varepsilon_{1}\right)+\left(\varepsilon_{4}-\varepsilon_{3}\right)=90^{\circ}+\alpha_{2}-\alpha_{1}-\varepsilon_{4} \\
= & 90^{\circ}+\alpha_{2}-\alpha_{1} \\
& -\sin ^{-1}\left(n_{2} \sin \left(\alpha_{2}-\sin ^{-1}\left(\frac{n_{1}}{n_{2}} \sin \left(\alpha_{1}\right)\right)\right)\right) .
\end{aligned}
$$

The flux's converging condition fixes the output angle $\beta_{n(\lambda)}$ and consequently the design parameters $\alpha_{1}, \alpha_{2}$ of the lens profile.

Due to the converging condition and assuming the width of the lens negligible compared to the focal distance $f$, the output angle is determined, based on Figure 5:

$$
\beta_{n(\lambda)} \cong \tan ^{-1}\left(\frac{f}{X_{n}}\right)
$$

Finally the design parameters for each profile's segment $\left(\alpha_{n 1}, \alpha_{n 2}\right)$ can be calculated:

$$
\begin{aligned}
\beta_{n}= & 90^{\circ}+\alpha_{n 2}-\alpha_{n 1} \\
& -\sin ^{-1}\left(n_{2} \sin \left(\alpha_{n 2}-\sin ^{-1}\left(\frac{n_{1}}{n_{2}} \sin \left(\alpha_{n 1}\right)\right)\right)\right) \\
= & \tan ^{-1}\left(\frac{f}{X_{n}}\right) .
\end{aligned}
$$

From the equation above (8), selecting an appropriate operation wavelength range $\left(\lambda_{1}=350 \mathrm{~nm}, \lambda_{2}=1000 \mathrm{~nm}\right)$ that sets the value of the refractive index $n_{1}$ and $n_{2}$ (of each material) at each wavelength, the achromatic lens profile whose behavior will be tested in subsequent simulations can be determined.

The wavelength range mentioned above is limited fundamentally by three factors.

(i) The first and most constraining one is obviously the solar emission spectrum which determines a minimum wavelength value for radiation at sea level around $300 \mathrm{~nm}$.

(ii) On the other hand the threshold determined by minimum radiation photon energy is able to stimulate materials commonly used for photovoltaic cells manufacture. In the case of silicon the cut-off wavelength is $1100 \mathrm{~nm}$ (equivalent to $1.2 \mathrm{eV}$ energy) [16] and it determines the maximum of the wavelength range which is designed for the achromatic doublet.

(iii) It must be also taken into account that the wavelength range selected determines the maximum linear chromatic aberration (LCA) within the operation range that corresponds to the difference of focal distances:

$$
\mathrm{LCA}=f\left(\lambda_{1}\right)-f\left(\lambda_{2}\right) .
$$

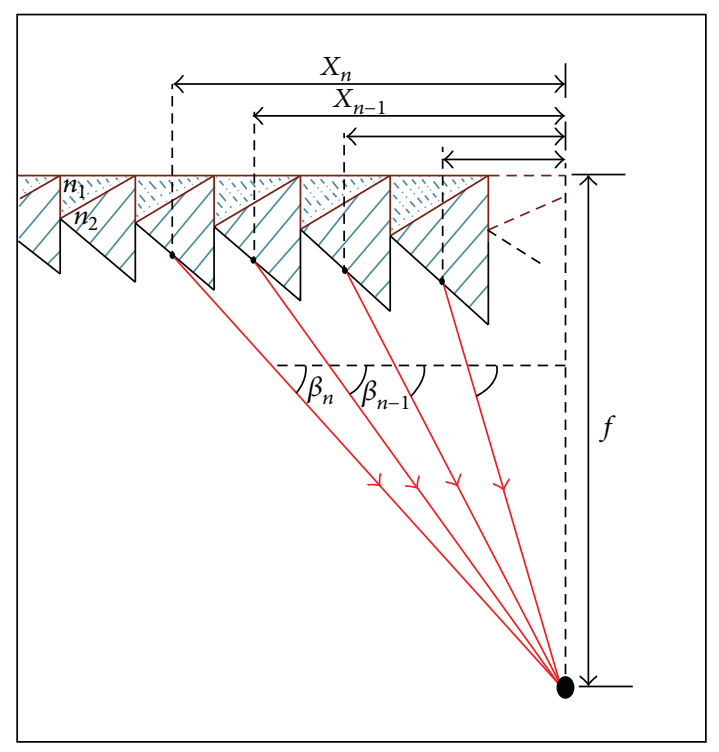

Figure 5: Output angle resolution.

\section{Results}

3.1. Standard Fresnel Lens versus Achromatic Design. As a first step the study of the incident light falling onto secondary's entry surface is analyzed, taking into account the efficiency, flux uniformity, and thrown area. The employed lens will be a circular Fresnel or achromatic lens of which focal length is $155 \mathrm{~mm}$ and the radio size is $R=120 \mathrm{~mm}$.

The distinction between the two achromatic lenses analyzed arises from its design and optimization process, of which main parameters are the prisms' steep size and angle (in terms of lens section) of each material of the achromatic doublet (PC \& PMMA), besides the location point to refer the output angle. Through this process the best structural pattern among the multiple possible configurations is reached. The optimization process is carried out for each prism of the lens and consequently applied to its annular sections.

The selection criterion of the two final achromatic lenses designs was focused on achieving maximizing flux level while ensuring minimum chromatic dispersion.

The steep angle parameter has been selected between two options that have proven to be optimal, whether choose the maximum or average difference between the prism angles (PC-PMMA). The reference point concerns the point regard each prism where the output angle is considered. Taking into account these different design strategies a set of achromatic lens are designed and simulated. The behavior of two achromatic lens designs (the best two configurations obtained by the parametrical development) has been compared versus a standard Fresnel lens of similar focal length and size. In Figure 6 the irradiance maps are shown for studied case at normal incidence considered for standard Fresnel and the best achromatic design.

As it can be seen comparing Figures 6(a) and 6(b) lighted area on secondary entry surface is much more reduced through the achromatic Fresnel lens. Computer optimized 
Fresnel lens
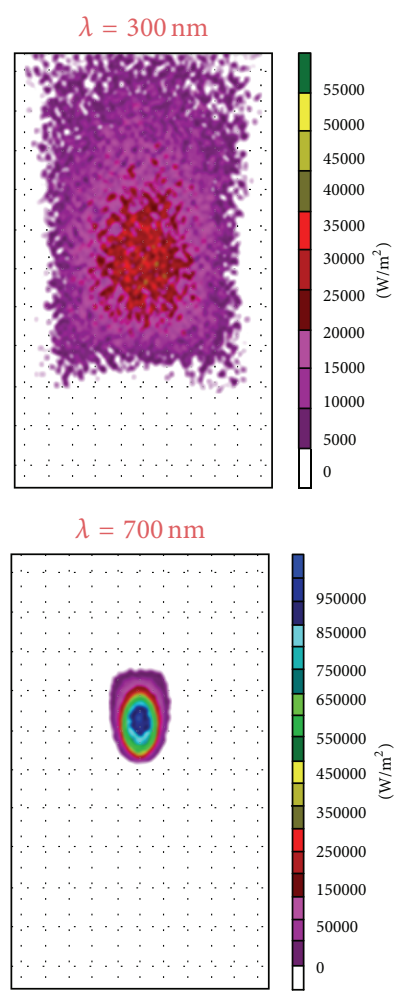

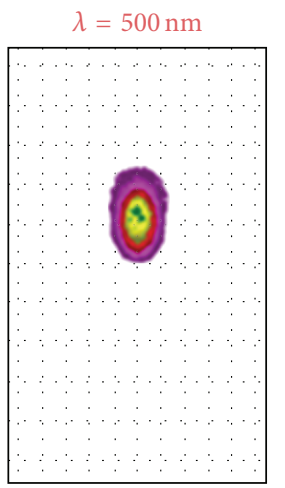

$\lambda=1000 \mathrm{~nm}$

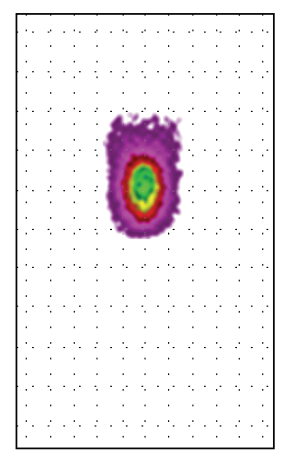

(a)
Achromatic lens 1
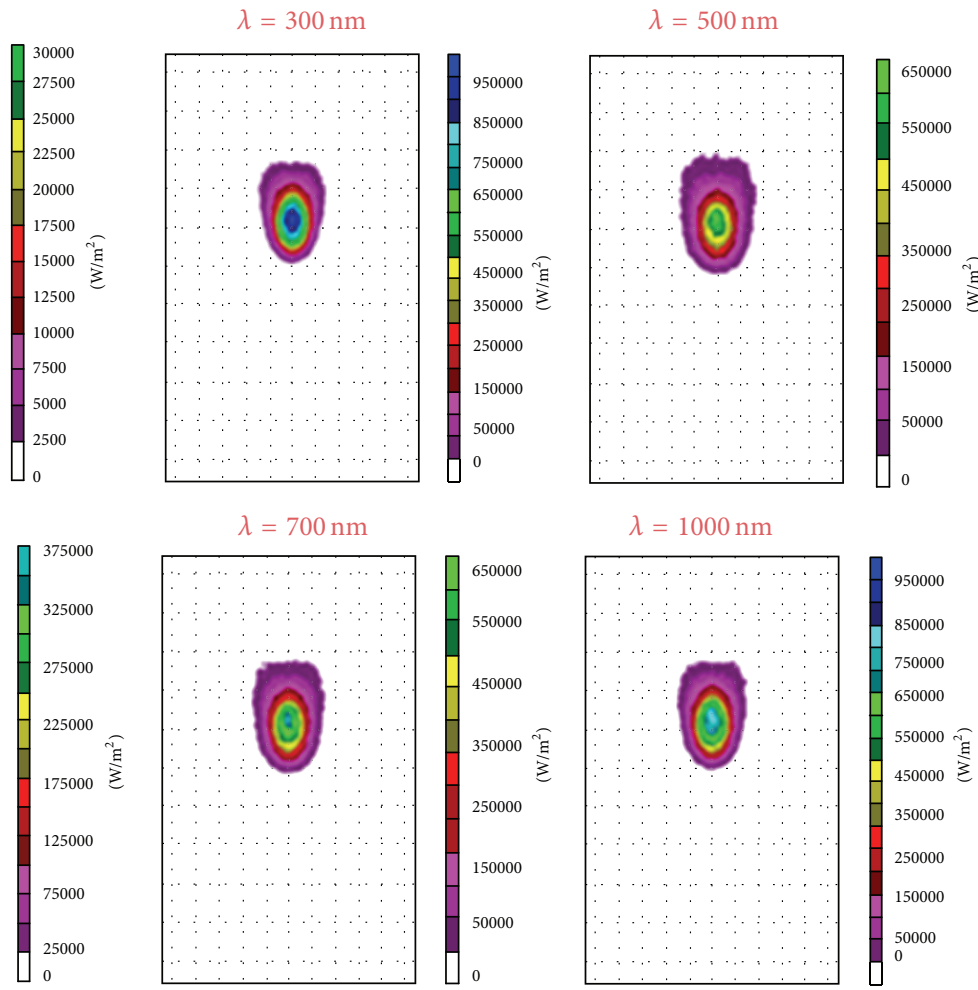

$\lambda=1000 \mathrm{~nm}$

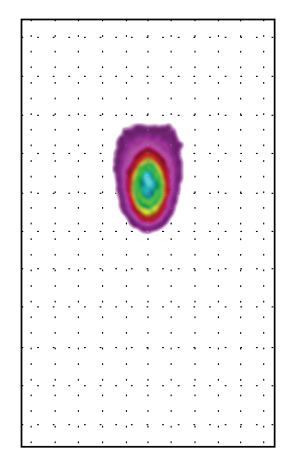

950000
850000
750000
650000
550000
450000
350000
250000
150000
50000
0

(b)

FIGURE 6: Irradiance maps on entry surface of secondary optics. Fresnel versus achromatic lens 1.

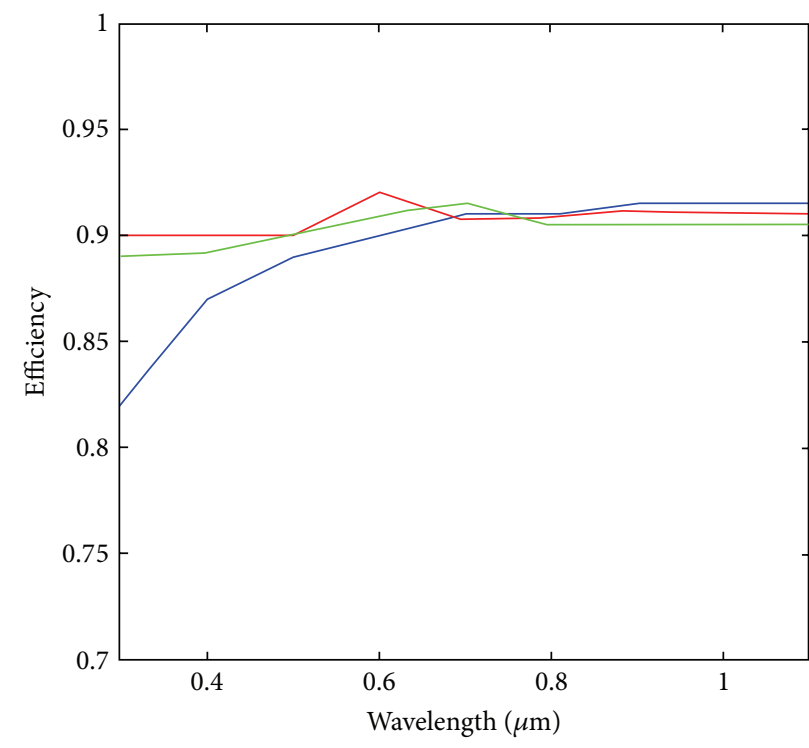

- Fresnel lens

Achromatic lens 1

- Achromatic lens 2

(a) Efficiency versus wavelength. Incidence angle $0.4^{\circ}$

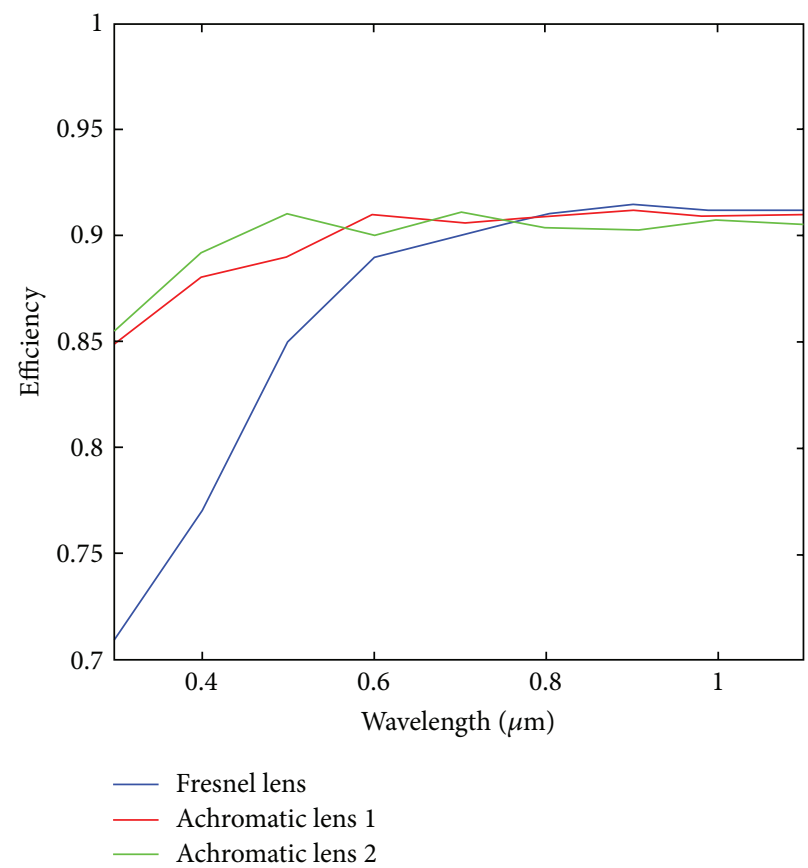

(b) Efficiency versus wavelength. Incidence angle $1^{\circ}$

FIGURE 7: Efficiency of achromatic and standard Fresnel lenses for $0.4^{\circ}$ and $1^{\circ}$ incidence. 


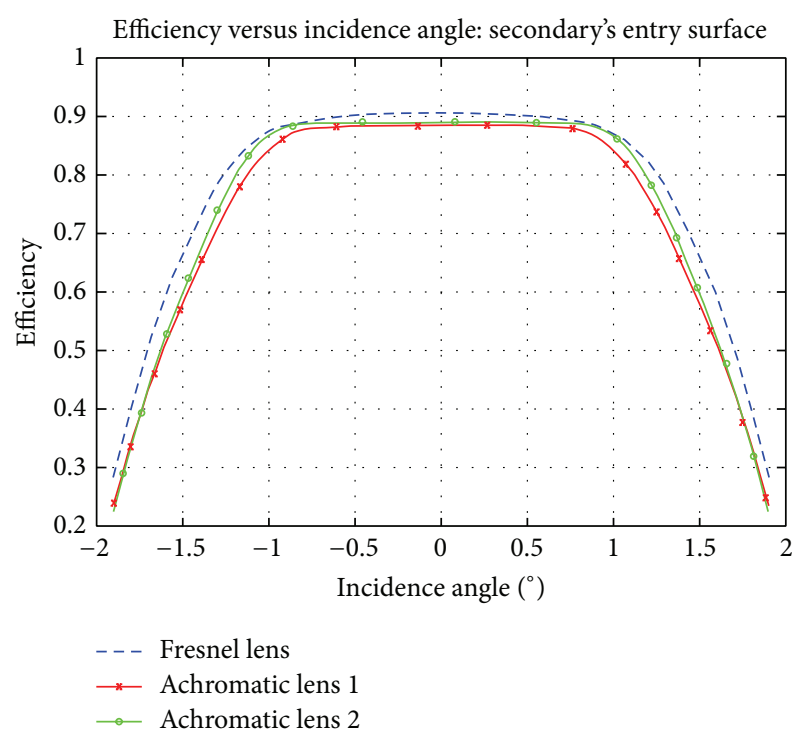

FIGURE 8: Pondered efficiency for whole spectra (ASTM 1) of solar light at different incidence angles.

designs minimize aberrations, yielding smaller focused spot sizes; it could permit us to use more compact secondary optics and therefore a smaller photocell.

System efficiency, $\eta$, defined here as the ratio of the luminous flux, $\Phi$, received by the input surface of the secondary optics to the total flux reaching the Fresnel primary lens, has been also analyzed:

$$
\eta=\frac{\Phi_{\text {secondary-input }}}{\Phi_{\text {lens surface }}}
$$

To evaluate the effect of achromatic aberrations in real conditions, not only the spectral range of the optical system but also the influence of the light's incidence angle is necessary to change the beam direction before focusing on the corresponding lens. Thus it is possible to have an idea of the deflection of light effect on the system, which is a common circumstance regarding concentrating and lighting solar systems where the source position is variable.

The efficiency of these three lenses (one standard and two achromatic) configuration, shown in Figure 7, is almost equivalent although the performance of the conventional Fresnel lens falls within a range of short wavelength, and this is particularly noticeable as the incidence angle increases, when greatest losses in Fresnel lens behavior are perceived. This effect is higher as the incidence angle grows, although the stabilization level reached around $750 \mathrm{~nm}$, regardless the the value $\left(0.4^{\circ}\right.$ or $\left.1^{\circ}\right)$ of incidence angle. This drift is caused by the chromatic dispersion effect, accentuated with a high incident angle, while the achromatic design keeps the focus for a broader range of wavelengths and has increased angular acceptance.

In order to evaluate the real effect of achromatic aberrations we have considered the importance of each wavelength taking into account the ASTM1 (American Society for Testing

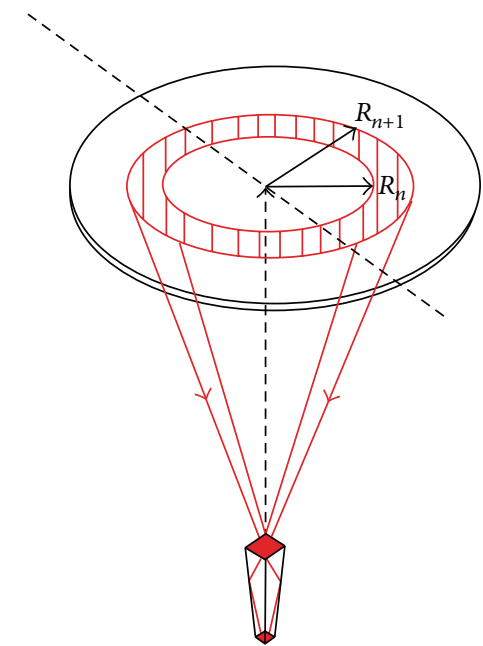

FIGURE 9: Ray tracing scheme of incident light ring of variable radius.

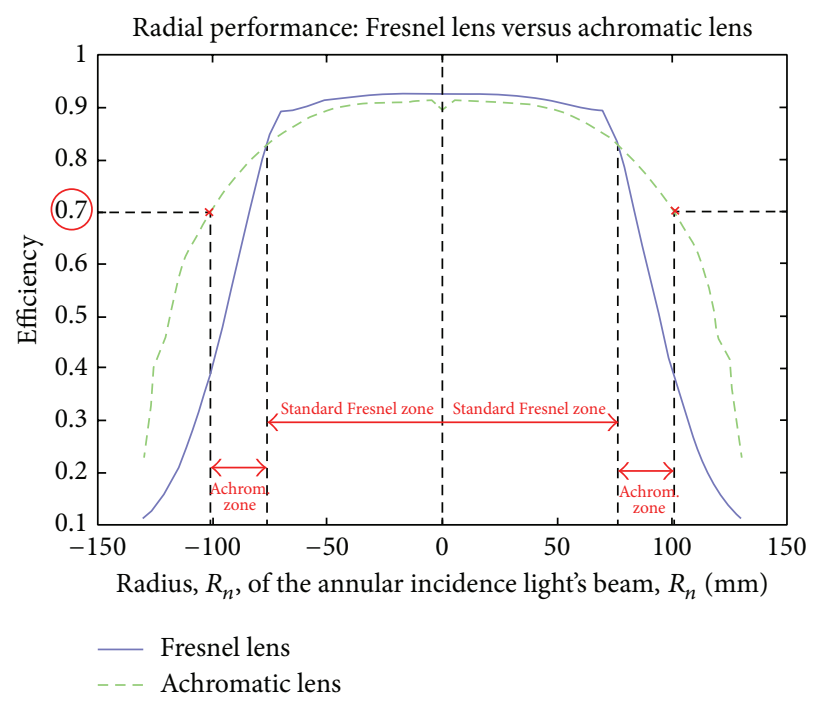

FIGURE 10: Efficiency of the achromatic lens versus annular incidence's radius.

and Materials (ASTM) Terrestrial Reference Spectra [17]) spectral distribution of sunlight.

As seen in Figure 8, the behavior of standard Fresnel lens (for selected numeric aperture, lens, and secondary optics size) is slightly better than achromatic design, a foreseeable result considering Fresnel lenses among nowadays solutions in the PV market. With the goal to obtain the best design it is necessary to know the minimal aperture from which the achromatic lens becomes better than the Fresnel one.

3.2. Optimized Fresnel Achromatic Design. Based on previous results standard Fresnel design probes to be an efficient enough solution as long as numerical aperture is limited; however if high concentration ratio is mandatory, then it is enforced to increase numerical aperture; in this case chromatic aberrations should be taken into account. Throughout 


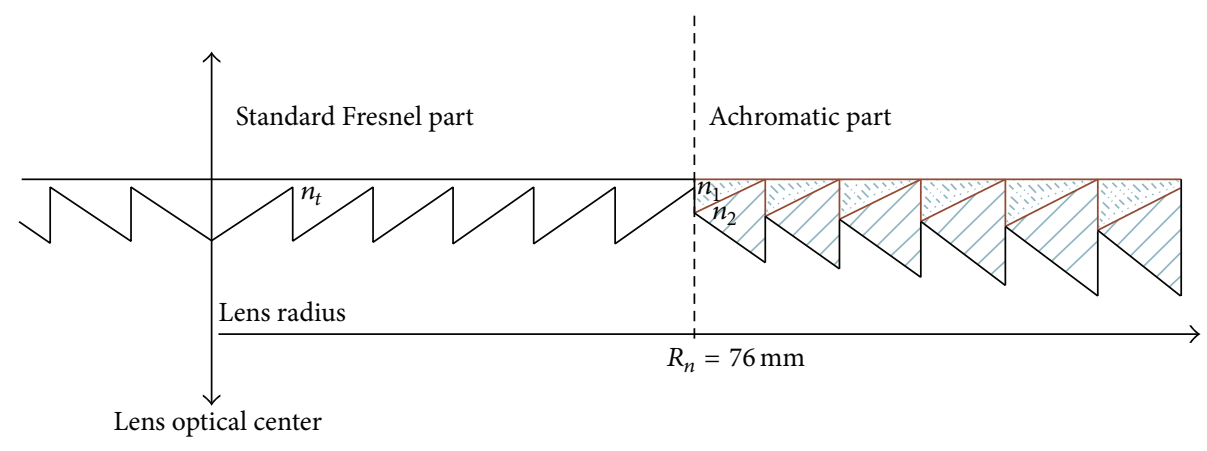

FIGURE 11: Hybrid lens section scheme.

TABLE 1: Achromatic lenses and distinguishing parameters.

\begin{tabular}{lcc}
\hline Design parameters & Achromatic lens 1 & Achromatic lens 2 \\
\hline Steep size $(\mathrm{mm})$ & 0.5 & 0.5 \\
Steep angle $\left(^{\circ}\right)$ & $\begin{array}{c}\text { Minimum difference } \\
\text { (PC-PMMA) }\end{array}$ & $\begin{array}{c}\text { Average difference } \\
\text { (PC-PMMA) }\end{array}$ \\
Reference point & Edge of prism step & Middle of prism step \\
\hline
\end{tabular}

this section an improved lens design has been developed combining the best of each typology (standard or achromatic design).

To evaluate the radial performance of the Fresnel lens versus both achromatic designs (Table 1 ) we have developed a series of simulations modifying the radius $\left(R_{n}, R_{n+1}\right)$ of a normal incidence light ring as seen in Figure 9.

To ensure that the concentration ratio remains constant the incident ring must maintain its area in each ray tracing interaction, so that flow per ring is maintained constant and thus the comparison, in terms of concentration, is relevant. Therefore the radius of the luminous disk follows next equation:

$$
R_{n}=\sqrt{n} R_{1} \Longrightarrow \Delta R=\pi\left(R_{n+1}^{2}-R_{n}^{2}\right)=\pi R_{1}^{2} .
$$

This study confirms that the achromatic design has advantages over the standard Fresnel lens as the light falls in the outer area. Figure 10 determines the radial boundary between the two designs for achromatic lens which shows superior performance to the standard Fresnel lens.

Figure 10 notes that standard Fresnel lens has higher efficiency until $R_{n}=76 \mathrm{~mm}$, that is, 0.23 N.A (numerical aperture). If the lower efficiency level of the lens is set as $70 \%$, then a hybrid Fresnel lens design is obtained by combining a central part based on standard Fresnel profile together with an achromatic design for the outer area up to a radius limit of $101 \mathrm{~mm}$ (Figure 11). This innovative design involves a standard part representing a $56.7 \%$ of the whole surface and an achromatic one that represents the $43.3 \%$ of the total aperture.

The improved performance of the hybrid achromatic lens compared to Fresnel conventional design is evidenced as the working area increases and can be analyzed in greater detail through a study of concentration provided by the lens

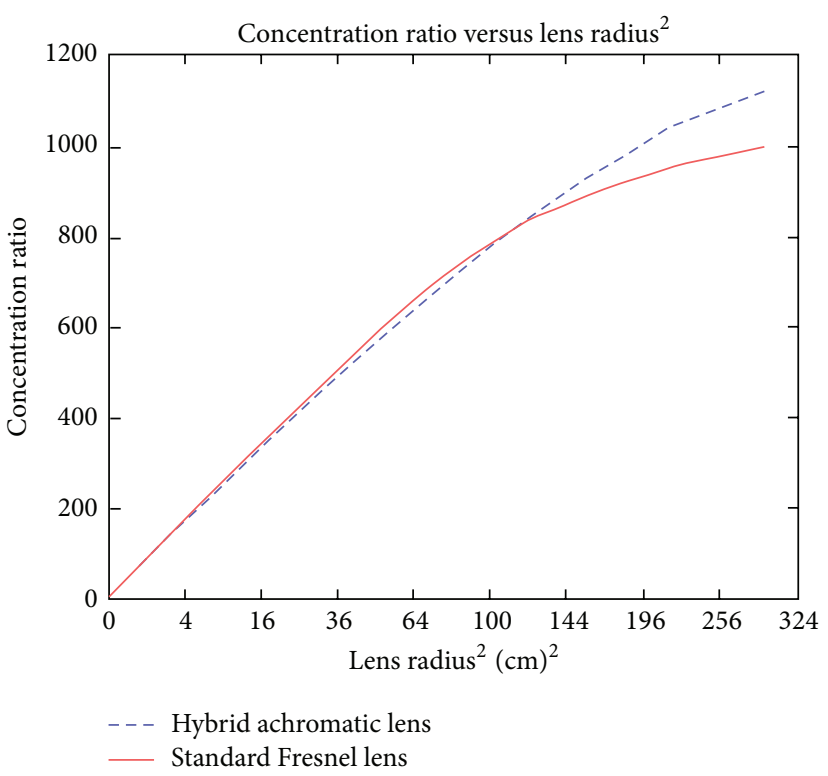

FIGURE 12: Concentration ratio versus lens square radius.

versus the squared radius. The concentration ratio has been calculated in the secondary optics output surface (photocell) for the whole spectrum selected:

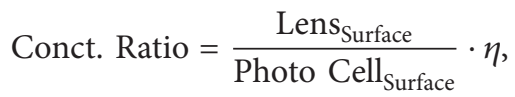

where $\eta$ is the efficiency regarding the incidence flux at the photocell.

It is verified that the hybrid achromatic lens offers advantages as the square of the radius increases, as shown in Figure 12, reaching concentration levels of above 1000 suns that hardly provide a conventional Fresnel lens.

Finally the performance of hybrid lens design versus pure achromatic and Fresnel lens is shown. A high efficient hybrid lens has been achieved combining the favorable areas of each design leading to a concentration system for increased stability against variation of the angle of incidence and higher concentration ratio.

The superior behavior for the hybrid lens design can be appreciated, especially compared to the Fresnel one; 


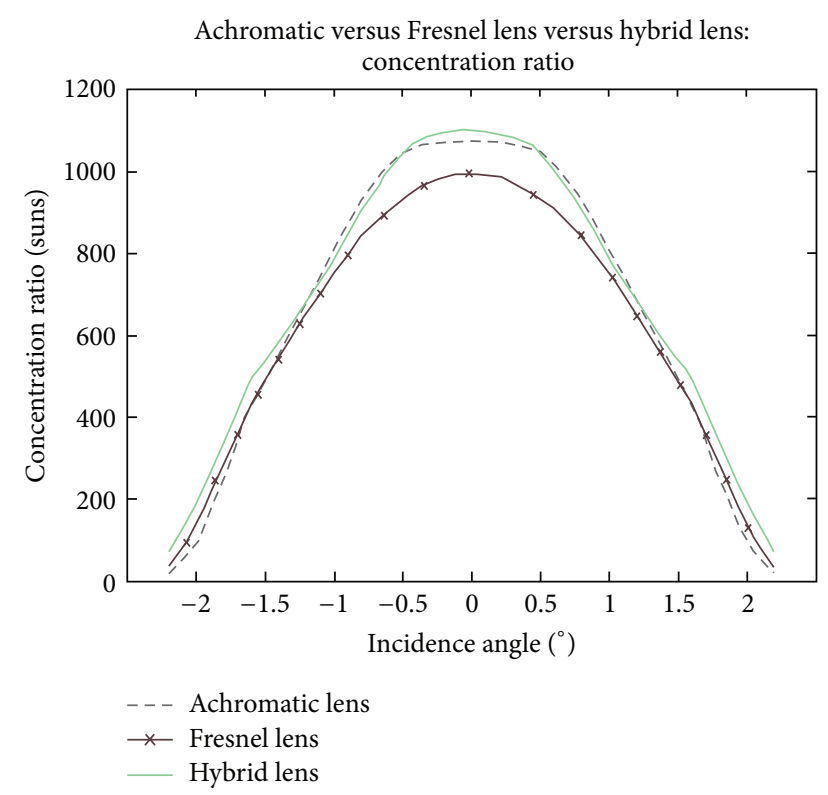

FIgURE 13: Concentration ratio versus incidence angle. Hybrid, achromatic, and Fresnel lens.

this performance is quite notable for relatively low incidence angles (less than one degree) where it reaches an $18 \%$ of improvement. This results can be observed in Figure 13. As the incidence angle increases the lens concentration profiles are homogenized but hybrid lens maintains a better performance compared to other designs, showing a $9 \%$ of difference at $1.5^{\circ}$ of incidence angle.

\section{Conclusions}

A new parametric design method for Fresnel achromatic doublets has been presented based on Snell equations and adaptable for different source's spectral and incidence characteristics. This method provides a high capacity to design this type of lenses and offers great versatility due to its multiple degrees of freedom. Through this parametric design, a novel type of achromatic lens has been developed consisting of a PC and PMMA Fresnel doublet which offers advantages in terms of efficiency (over $85 \%$ for the whole wavelength operation range) and concentration (above 1000 suns) relative to the standard Fresnel lenses; these features are manifest from a numerical aperture's threshold of 0.23 and become remarkable as the dimensions of the concentration lens increase.

Through a process of optical design and computer aided simulation, a hybrid achromatic lens has been achieved; this type of lens combines the advantages of standard Fresnel lenses combining these with the most useful features of the achromatic lens. Throughout this procedure several solutions have been compared in terms of efficiency, flux distribution, concentration factor, and dependence on wavelength or incidence angle. Thus it has achieved a new lens design, with high numerical aperture, capable of a high degree of efficiency higher than standard Fresnel lens, and increasing the stability against the angle of incidence and spectral components of incident flux.
This improved design can be very effective as primary optics for photovoltaic cell systems maximizing the capture area of the focusing lens without sacrificing the efficiency and decreasing the size of the spot and thereby ensuring a higher level of concentration is also maintained for a longer interval of time, as the response of the lens is also more stable against changes in the incidence of the beam. Specifically, through this novel hybrid lens design, a concentration level of $18 \%$ higher than the standard Fresnel lens has been obtained.

\section{Conflict of Interests}

The authors declare that there is no conflict of interests regarding the publication of this paper.

\section{References}

[1] V. N. Mahajan, Optical Imaging and Aberrations: Ray Geometrical Optics, SPIE Optical Engineering Press, Bellingham, Wash, USA, 1998.

[2] E. Lorenzo, "Chromatic aberration effect on solar energy systems using Fresnel lenses," Applied Optics, vol. 20, no. 21, pp. 3729-3732, 1981.

[3] E. Hecht, Optics, Addison-Wesley, Toronto, Canada, 4th edition, 2001.

[4] G. K. Skinner, "Design and imaging performance of achromatic diffractive-refractive X-ray and gamma-ray Fresnel lenses," Applied Optics, vol. 43, no. 25, pp. 4845-4853, 2004.

[5] Y. W. Zhang, C. S. Ih, H. F. Yan, and M. J. Chang, "Photovoltaic concentrator using a holographic optical element," Applied Optics, vol. 27, no. 16, pp. 3556-3560, 1988.

[6] J. Strong, "Achromatic doublet lenses for infrared radiation," Applied Optics, vol. 10, no. 6, pp. 1439-1443, 1971.

[7] E. M. Kritchman, A. A. Friesem, and G. Yekutieli, "Highly concentrating Fresnel lenses," Applied Optics, vol. 18, no. 15, pp. 2688-2695, 1979.

[8] F. Languy, C. Lenaerts, J. Loicq, T. Thibert, and S. Habraken, "Performance of solar concentrator made of an achromatic Fresnel doublet measured with a continuous solar simulator and comparison with a singlet," Solar Energy Materials and Solar Cells, vol. 109, pp. 70-76, 2013.

[9] Fresnel lens brochure of the Fresnel Technologies, http://www .fresneltech.com/materials.html.

[10] F. Languy, C. Lenaerts, J. Loicq, and S. Habraken, "Experimental results of hybrid and refractive achromatic doublets made of PC and PMMA," in Proceedings of the Renewable Energy and the Environment Optics and Photonics Congress, Optical Society of America, Eindhoven, The Netherlands, 2012.

[11] M. Z. Shvarts, V. M. Andreev, V. S. Gorohov et al., "Flatplate Fresnel lenses with improved concentrating capabilities: designing, manufacturing and testing," in Proceedings of the 33rd IEEE Photovoltaic Specialists Conference (PVSC '08), pp. 16, San Diego, Calif, USA, 2008.

[12] A. Davis, K. Levesque, and S. Wilt, "Prism peak rounding in injection molded Fresnel lens solar concentrators," in Diamond Turning and Polymer Optics, C. Ghio, Ed., TD07, Optifab, 2011.

[13] K. Maekawa, Y. Hachisu, H. Ohmori et al., "JEM-EUSO lens manufacturing," in Proceedings of the 31st International Cosmic Ray Conference (ICRC '09), Lodz, Poland, 2009. 
[14] M. Victoria, C. Domínguez, I. Antón, and G. Sala, "Comparative analysis of different secondary optical elements for aspheric primary lenses," Optics Express, vol. 17, no. 8, pp. 6487-6492, 2009.

[15] M. Laikin, Lens Design, CRC Press, Toronto, Canada, 4th edition, 1991.

[16] G. M. Masters, Renewable and Efficient Electric Power Systems, John Wiley \& Sons, New York, NY, USA, 2004.

[17] American Society for Testing and Materials (ASTM) Terrestrial Reference Spectra, http://rredc.nrel.gov/solar/spectra/am1.5/. 

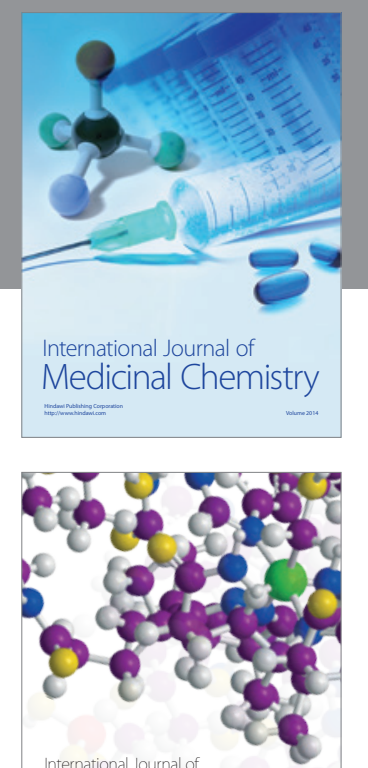

\section{Carbohydrate} Chemistry

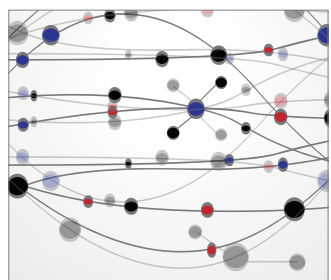

The Scientific World Journal
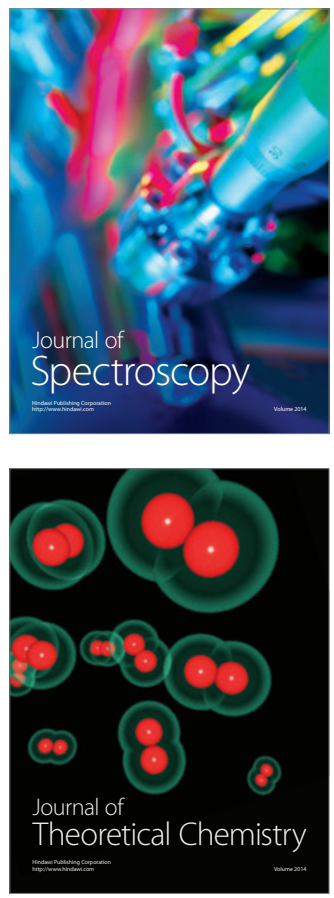
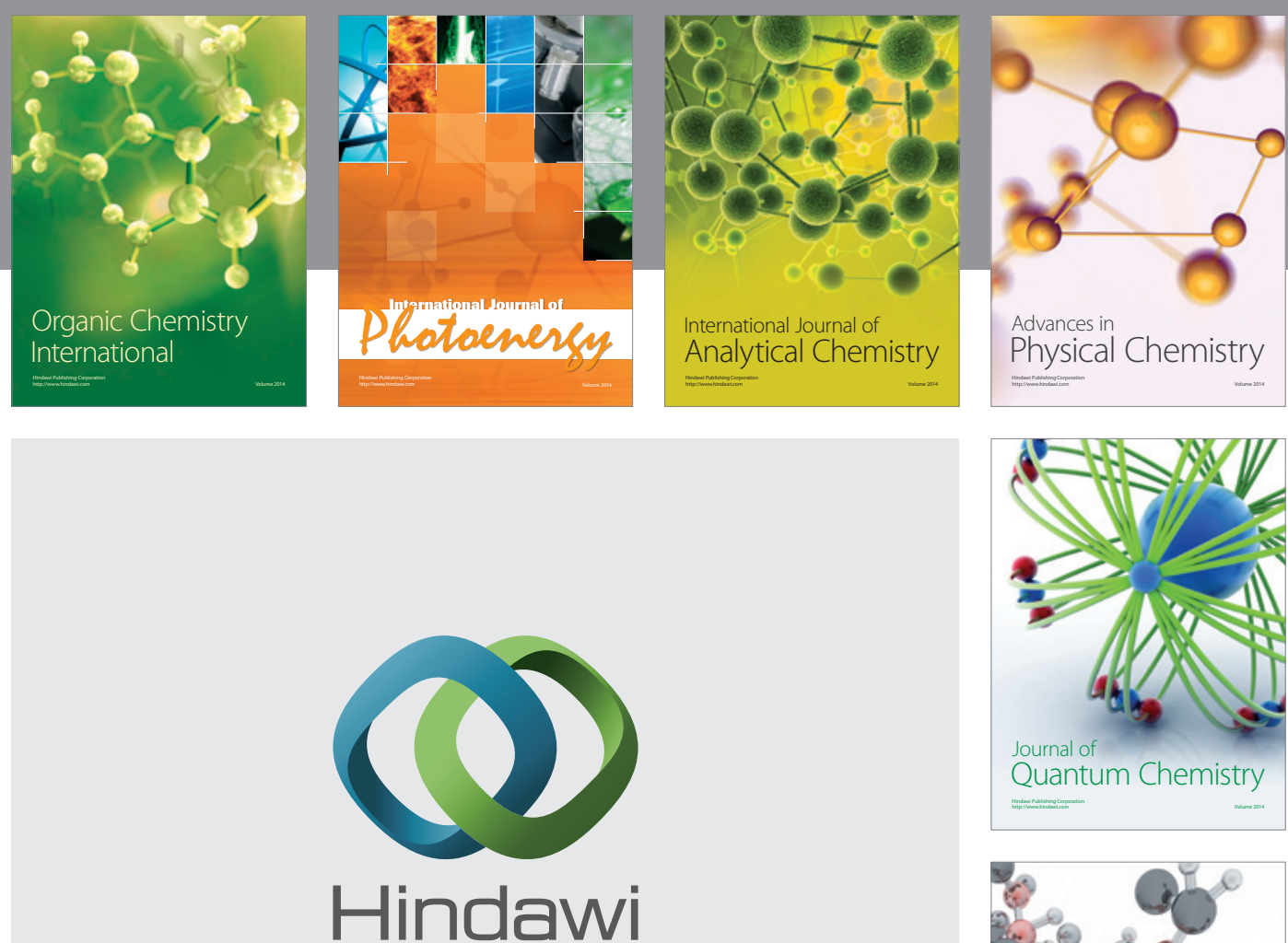

Submit your manuscripts at

http://www.hindawi.com

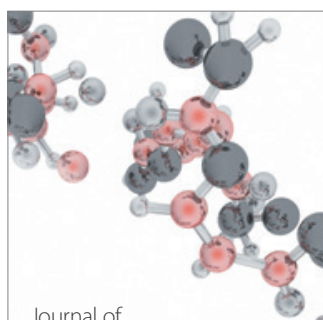

Analytical Methods

in Chemistry

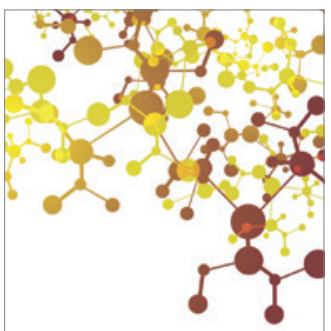

Journal of

Applied Chemistry

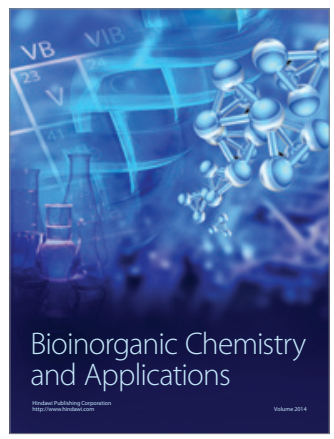

Inorganic Chemistry
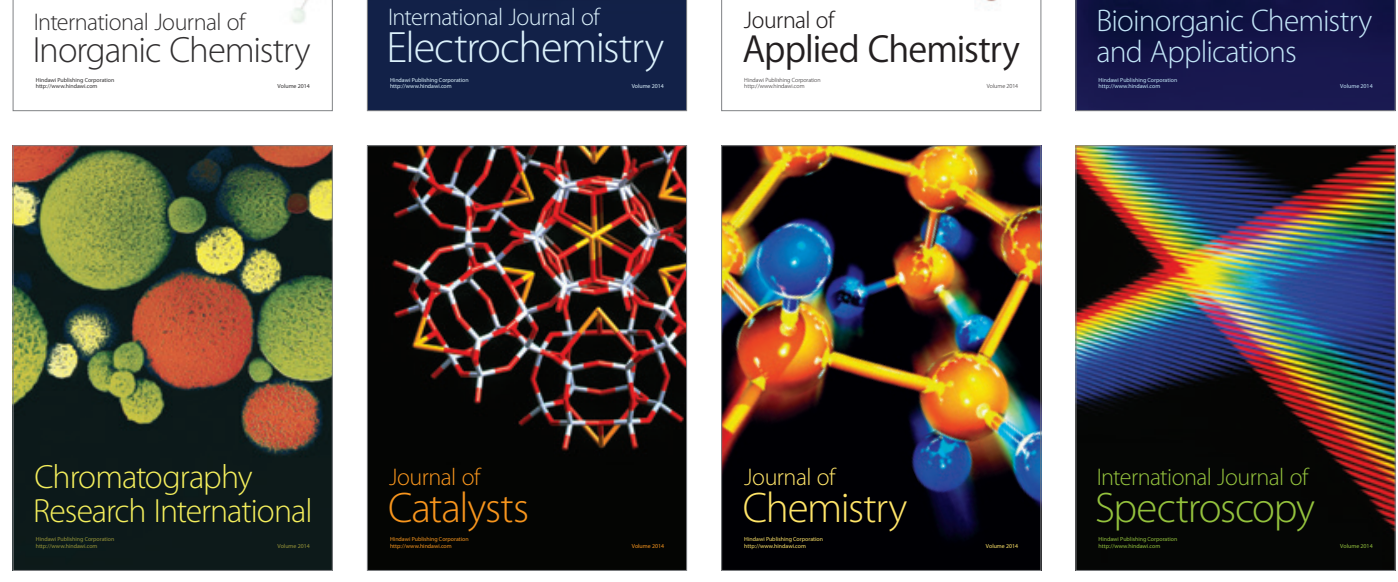